\title{
Neue Bücher aus der Weiterbildungsforschung
}

\section{Ewa Przybylska · Petra H. Steiner • Ekkehard Nuissl • Lorenz Lassnigg}

Online publiziert: 4. Juni 2018

(C) Der/die Autor(en) 2018

\section{Rezensionen zu:}

Elke E. Theile (2017). Die deutsch-polnische Beziehungsgeschichte im Fokus der Erwachsenenbildung. Frankfurt/Main: Wochenschau Verlag. 552 Seiten, 59,90€, ISBN 978-3-7344-0516-7

Annabel Jenner (2018). Lernen von Mitarbeitenden und Organisationen als Wechselverhältnis. Eine Studie zu kooperativen Bildungsarrangements im Feld der Weiterbildung. Wiesbaden: Springer. 270 Seiten, 46,25€, ISBN 978-3-658-19453-6 Sabine Lauber-Pohle (2017). Soziale Netzwerkbildung und Online-Lernen. Wiesbaden: Springer. 260 Seiten, 44,99€, ISBN 978-3-658-19264-8

Walter Schöni (2017). Bildungswertschöpfung: Zur politischen Ökonomie der berufsorientierten Weiterbildung. Bern: Hep. 284 Seiten, 32,00€ ISBN-Print: 978-30355-0733-1

E. Przybylska $(\square)$

Universität Warszawa, Warschau, Polen

E-Mail: p-nfed@wp.pl

P. H. Steiner

Weiterbildungsakademie Österreich (wba), Wien, Österreich

E-Mail: petra.steiner@wba.or.at

E. Nuissl

Universität Florenz, Florenz, Italien

E-Mail: nuiss1@die-bonn.de

L. Lassnigg

Institut für Höhere Studien, Wien, Österreich

E-Mail: lassnigg@ihs.ac.at 


\section{Ewa Przybylska: Elke E. Theile (2017). Die deutsch-polnische Beziehungsgeschichte im Fokus der Erwachsenenbildung. Frankfurt/ Main: Wochenschau Verlag. 552 Seiten, 59,90€, ISBN 978-3-7344- 0516-7}

Die Autorin beschäftigt sich mit den Herausforderungen der Erwachsenenbildung in Bezug auf die Aufarbeitung und Vermittlung der deutsch-polnischen Beziehungsgeschichte. Der Leitgedanke des Buches - „Aus der Geschichte lernen“ (S. 7) sollte eigentlich als selbstverständliche Prämisse erscheinen, dennoch gelingt es in der Realität längst nicht immer, ihm zu folgen, geschweige denn, ihn in die Praxis umzusetzen.

Das Buch erschien im Sommer 2017, zum Zeitpunkt des potenziellen Scheiterns eines die politischen und wirtschaftlichen Beziehungen betreffenden Modells von auf Gegenseitigkeit, gemeinsamen Interessen und einer strategischen Partnerschaft beruhender deutsch-polnischer Beziehungen. Im Zuge einer realen Bedrohung des Verlustes von seit dem demokratischen Umbruch erzielten Fortschritten gewinnt seither die Forderung an Stärke, wonach die Zivilgesellschaften beider Länder mehr Verantwortung für die Erhaltung und Entwicklung des gegenseitigen Vertrauens und der Zusammenarbeit zu übernehmen hätten. „Für die historisch-politische Erwachsenenbildung sind die Aufarbeitung der deutsch-polnischen Beziehungsgeschichte und die Analyse der Erinnerungskultur eine Notwendigkeit, um friedenspädagogische Lösungswege für ein besseres gegenseitiges Kennen- und Verstehen-Lernen aufzuzeigen“, konstatiert die Autorin (S. 8). Folglich richtet sich der Blickwinkel des Buches ,,auf die historisch-politischen Beziehungsmuster“ in erwachsenenpädagogischer Bildungspraxis (S. $7 \mathrm{f}$.).

Gewiss hält die „Konjunktur des Gedächtnisses“ an, erfreut sich die Erinnerungskultur als interdisziplinärer Forschungsgegenstand beständiger Popularität, doch nie zuvor wurde das Thema im Rahmen eines kohärenten wissenschaftlichen Ansatzes in der Erwachsenenbildung verortet, wie im Falle der Forschungsstudie von Elke E. Theile. Die Autorin interessiert sich weniger für die Vergangenheit an sich als vielmehr für ihr gesellschaftliches Bild im Bewusstsein der verschiedenen Akteure und in Relation zum Prozess gesellschaftlicher Erinnerung, der sich im sozialen Gedächtnis niederschlägt.

Das äußerst ,zeitgemäße“ literarisch-wissenschaftliche Werk weicht in signifikanter Weise durch seine besondere Struktur vom in der Literatur der Erwachsenenbildung gängigen Kanon ab. Im ersten und umfassendsten Kapitel präsentiert Elke E. Theile auf über dreihundert Seiten die tausendjährige Geschichte der deutschpolnischen Beziehungen. Sie geht dabei sowohl auf Ereignisse, gegenseitige Beeinflussungen und Momente bereichernder Nachbarschaft als auch auf Konflikte und Katastrophen ein, die bis heute Populisten und Rechtsradikalen dazu dienen, gegenseitige Feindseligkeit zu nähren. Der geschichtliche Teil des Buches bildet eine „Informationsgrundlage und Voraussetzung für die Analyse der Erinnerungskulturen als historisch-politische Reflexionsleistung der erinnerungskulturorientierten ,sinnorientierten“ Erwachsenenpädagogik“" (S. 8). 
Im Vordergrund des zweiten Kapitels stehen Kategorien, wie die ,autonome Urteils- und Entscheidungsfähigkeit des Erwachsenen“, „,das kollektive und individuelle Gedächtnis“, „Erinnern und Gedenken“, „Sinnzusammenhang der öffentlichen politischen und der privaten Erinnerung“, „Wahrnehmung“, „Deutung“ u.v.a.m. „Die ewachsenenbildungswissenschafliche Analyse der Erinnerungskultur ist auf der Grundlage historischer und politischer Erkenntnisse auch auf Deutungs- und Erklärungselemente verschiedener Nachbardisziplinen, z. B. der Kultur-, Sozial- und Verhaltenswissenschaft angewiesen“, resümiert die Autorin (S. 386). Demnach entwirft sie einen ,intersystematisch-handlungstheoretischen Kommunikationsansatz“ (S. 424) zur Unterstützung der Aufarbeitung des bilateralen Beziehungsverhältnisses mit seinen Geschichtsbildern und Erinnerungskulturen.

Im dritten Teil des Buches, der die Erinnerungskultur zur deutsch-polnischen Kultur auf den Ebenen der Forschung und Praxis in den verschiedenen Lernorten in den Blick nimmt, vermittelt die Autorin gut verständlich und praxisnah die Möglichkeiten der Erinnerungskulturarbeit zur deutsch-polnischen Beziehungsgeschichte und beleuchtet anhand von Beispielen (Lernprozesse in Gedenkstätten, Städtepartnerschaften, Deutsch-Polnische Gesellschaften) die „Brückenfunktion“ der Erwachsenenbildung im Bereich der historisch-politischen Aufklärung und Aufarbeitung.

$\mathrm{Zu}$ hoffen bleibt, dass politische Prozesse hier und dort nicht das Ende der Länderpartnerschaft herbeiführen und Polen und Deutsche nicht auf Pfade führen, vor denen uns die Geschichte warnt. Schon jetzt erscheint es, dass mit dem Schreiben der Zeitgeschichte befasste Wissenschaftler sich immer wieder veranlasst sehen werden, den antideutschen Phobien der Regierungspartei in Polen und populistischen Strategien beiderseits der Grenze, die historischen Relativismus und gefährliche Ressentiments gebrauchen, ihr Augenmerk zu widmen. Umso wichtiger und notwendiger ist dieses Buch, und sicher auch von Nutzen für all die in der historischkulturellen Erwachsenenbildung in Praxis und Wissenschaft Tätigen, denen Demokratie, Frieden und Dialog zwischen den Nachbarn am Herzen liegen. „Angesichts des Nationalkonservatismus und Rechtspopulismus in Europa“, betont Theile, ,ist für Deutsche und Polen [...] das ,Friedensobjekt Europa “ - in Erinnerung an die Katastrophen des 20. Jahrhunderts - ein besonderes Gut, dass es gemeinsam zu pflegen gilt" (S. 323).

\section{Petra H. Steiner: Annabel Jenner (2018). Lernen von Mitarbeitenden und Organisationen als Wechselverhältnis. Eine Studie zu kooperativen Bildungsarrangements im Feld der Weiterbildung. Wiesbaden: Springer. 270 Seiten, 46,25 $€$, ISBN 978-3-658-19453-6}

Anliegen des Buches ist es, zu klären, inwiefern das (individuelle) Lernen von Mitarbeitenden in Weiterbildungseinrichtungen zu organisationalem Lernen beiträgt. So lautet die Forschungsfrage: ,Welche kommunikativen Prozesse ermöglichen, das Lernen von Mitarbeitenden in Kooperationen in organisationales Lernen innerhalb der Herkunftsorganisation zu transformieren?" (S. 23) Die Autorin verortet sich wissenschaftlich in der Erwachsenenpädagogik. Sie bearbeitet die Frage anhand des Betrachtens von Organisationen, welche innerhalb von ,kooperativen Bildungsar- 
rangements“ aufeinandertreffen. Daraus ergibt sich auch die zusätzliche Verortung der Forschungsarbeit in der Kooperations- und Netzwerkforschung. Dabei kann die Studie auf einen bereits vorhandenen Forschungsstand aufbauen, der durch die vorliegende Arbeit eine neue Nuancierung erfährt: So zeigen sich teilweise systemtheoretische Bezüge, was auch spezifische Folgen für den Blickwinkel der Forschenden zeitigt. Die verwendete Sprache fordert vom Lesenden hohe Konzentration, gleichzeitig leistet sie wohl das, was nach Luckmann jede sozialwissenschaftliche Forschung zu leisten hat: Forschende schaffen demnach andere Lesarten für soziale Wirklichkeiten und können Muster und Strukturen, die hinter sozialen Erscheinungen liegen, benennen, indem sie Distanz zu den Phänomenen einnehmen.

Beeindruckend sind das forschungslogisch disziplinierte Vorgehen und der hohe Anspruch der stringenten theoriebasierten Entfaltung aller Schritte der Erhebungsarbeit. Die Autorin versäumt es nicht, die Schritte genau zu beschreiben und nimmt so die interessierten Lesenden mit auf die Reise durch ein anforderungsreiches Forschungsprojekt. Man liest über das aufwändige Vorgehen bei der Fallauswahl, erfährt, wie genau die systemtheoretische Fragemodellierung zustande kam, wie etwa der zu analysierende „Fall“ einer „Schnittstelle“ theoretisch konzipiert wurde und wie in der Folge das theoretische Konstrukt empirisch eingeholt und auch ein Stück weit die Ausgangsideen verändert wurden. In einem Teil der ,,metareflexiven Beobachtung“ des Forschungsprozesses berichtet die Autorin, wie sie sich zwischen Theorie und Empirie suchend hin- und herbewegen muss. Ein für die Weiterbildungsforschung fruchtbarer Gewinn aus dieser Arbeit dürfte in der theorie- und empiriebasierten ,,heuristischen Rahmung der empirischen Befunde“ (S. 213) für das Zusammenwirken von individuellem und organisatorischem Lernen in dem untersuchten Bereich liegen. Hier sind die Konzepte „Vor- und Rückbindung“ von Lernakten zwischen ,entsendeten“ Mitarbeitenden und Organisation hervorzuheben sowie die Systematisierung individueller Lernanlässe und die von der Organisation geleisteten „strukturgenerierenden Leitentscheidungen“, für welche eine inhaltliche Kategorisierung vorgelegt wird.

Die Ergebnisse der Forschungsarbeit sind dementsprechend stark auf die theoretische Weiterentwicklung abgestellt, v. a. Forschende der Weiterbildungsforschung im Bereich Organisationsforschung sowie Netzwerk- und Kooperationsforschung und Forschende mit starkem theoretischen Interesse finden in dem Buch die Darlegung eines wissenschaftlichen Prozesses, der sich in aller Transparenz zeigt. Die Brücke zwischen individuellem und organisationalem Lernen zu schließen, ist die Leistung, die bislang in dieser fundierten Weise wohl seltener erbracht wurde.

Inhaltliche Ergebnisse des Forschungsprojektes in ihrem Wert für die Praxis sind dagegen schwieriger herauszudestillieren, allenfalls ist es spannend, einen Einblick in das komplexe Ineinandergreifen individuellen Lernens und organisatorischer Operationen zu gewinnen. So zeigt sich etwa, dass Organisationen mitnichten nicht mit Lernanlässen umgehen, sondern im Gegenteil ein Arsenal an Möglichkeiten nutzen, um Lernanlässe wahrzunehmen - an vielen Stellen einer „Prozesskette“ werden Entscheidungen zugunsten oder zuungunsten einer Weiterverarbeitung der Irritation getroffen. Auch die Produktivität und Aktivität des Zurückweisens von neuen Lernanlässen, um Bestehendes aufrechtzuerhalten, gerät in den Blick. Der Anspruch an Organisationen wird von der Autorin dabei zukunftsweisend und recht ,,theorielas- 
tig“ formuliert, etwa: „In einer solchen Perspektive geht es darum, organisationsinterne Reflexionsgelegenheiten dafür zu schaffen, dass die Organisation ihr Vermögen zum Hören, Analysieren, Bewerten und Hervorbringen von Entscheidungen über eine handlungsorientierte Weiterverarbeitung selbst beobachtet" (S. 266).

Angesichts der gegenwärtigen Anforderungen an Organisationen der Weiterbildung, sich innerhalb verschiedenster gesellschaftlicher Felder zu positionieren, zu bewähren, weiterzuentwickeln und so gesellschaftsgestaltend zu wirken, kann die bearbeitete Frage als hoch relevant eingeschätzt werden.

\section{Ekkehard Nuissl: Sabine Lauber-Pohle (2017). Soziale Netzwerkbildung und Online-Lernen. Wiesbaden: Springer. 260 Seiten, 44,99€, ISBN 978-3-658-19264-8}

Man könnte meinen, es sei mittlerweile schon genug zum medial umgeformten Lehren und Lernen geforscht und geschrieben worden, sei es nun E-Learning, OnlineLearning oder Blended Learning. Und man könnte meinen, dass nun, da der „Hype“ um die Verwendung von Medien zur Gestaltung von Lehr-Lern-Prozessen abgeklungen ist, eine weitere Publikation zum Thema nur noch auf geringe Aufmerksamkeit zu stoßen vermag. Dies ist bei der publizierten Forschungsarbeit von Sabine LauberPohle nicht der Fall. In erster Linie liegt dies sicherlich daran, dass die Autorin das Medienthema mit dem Diskurs zu Netzwerken verbindet, der ja, aus gutem Grund, immer wieder aktualisiert und differenziert die sozialwissenschaftliche Forschung (und nicht nur diese) anregt und befruchtet.

Die Autorin stellt sich die Frage: ,Wie gestalten sich die Wechselwirkungen zwischen Online-Lernen und sozialer Netzwerkbildung?“ mit den ,,vertiefenden Teilfragen“: ,Welchen Einfluss nehmen soziale Netzwerke auf den Verlauf und die Gestaltung von Online-Lernen? Und wie wirkt sich Online-Lernen auf die die Lernenden umgebenden sozialen Netzwerke aus?“" (S. 14). Ein Blick also in beide Richtungen nicht nur mit dem Ziel, mehr über die entsprechenden Wirkungen und Zusammenhänge zu ermitteln, sondern damit auch Potential für Reflexionen zu Lernen und Beraten in der Erwachsenenbildung zu schaffen.

Eine dritte Größe zwischen Netzwerken und Online-Lernen bestimmt die Forschungsarbeit ganz wesentlich: die lernenden Subjekte. Die Autorin erkundet die beiden Pole ihrer Fragestellung aus der Sicht der Menschen, die online lernen und sich in sozialen Netzwerken bewegen. Und damit befindet sie sich im Zentrum der erwachsenenpädagogischen Forschung seit mehr als drei Dekaden: die Verortung des Lernens Erwachsener in deren sozialem Umfeld und vor ihrem biografischen Hintergrund.

Bei den Lernenden differenziert sie vor allem deren „Konstitutionsleistungen“. Mit diesen knüpft sie an die Kategorisierung von Kade und Seitter in deren Analyse von Funkkolleglernern (also noch vor dem Medienhype) an; die drei Konstitutionsleistungen sind die Herstellung der Lernbedingungen, die soziale Akzeptanz der Teilnahme und die biographische Passungsfähigkeit (S. 62 ff.). Mittels dieser Konstitutionsleistungen, so die Vermutung der Autorin, konturieren sich die Wechselbezie- 
hungen zwischen medialem Lernen und sozialem Netzwerk - auf je unterschiedliche Weise.

Die Vorgehensweise der Autorin ist komplex: Ihre Erkenntnisse bauen auf Interviews mit einem Sample von 18 Personen auf, die leitfadengestützt narrative und problemorientierte Elemente verbanden (S. 66ff.). Die akribisch transkribierten Interview-Daten wurden auch codiert und in drei Materialdurchgängen sequenzanalytisch interpretiert. Auf eine quantitative Auswertung, so die erfreuliche und erfreulich klare Reflexion der Autorin (S. 83 ff.) wurde aus nachvollziehbaren Gründen verzichtet.

Um über eine Sammlung von Fallstudien hinauszukommen, nahm die Autorin eine Typenbildung vor. Hier lag mit Sicherheit die schwierigste Herausforderung des empirischen Teils: Die Typen sollten ja jeweils Merkmale des sozialen Netzwerks als auch des Online-Lernens ausweisen. Hinsichtlich der Netzwerke verzeichnete die Autorin starke Ähnlichkeiten bei den erstellten „Netzwerkkarten“ etwa Bedeutungszuschreibungen bei Freunden und Familie oder in der Ausdifferenzierung und Verschränkung beruflicher Netzwerke; ähnliches galt auch für die Integration der Lerngruppe in das allgemeine und das berufliche Netzwerk. Für das Online-Lernen differenzierte die Autorin in der Typenbildung vor allem auf Lernmotivation und Lernanlass. Hinzu kamen personenbezogene Daten (Ausbildung, Biographie) und solche zu Gegenstand und Dauer des Online-Angebots (S. 115).

Die einzelnen Daten komprimierte die Autorin zu fünf Typen: Erkundung, Aufbau, Orientierung, Neuausrichtung und Verstetigung, die sie ausführlicher beschreibt (S. $119 \mathrm{ff}$.). Darauf aufbauend erläutert Lauber-Pohle jeweils typspezifisch zunächst den Umgang mit dem Online-Lernen hinsichtlich Motivation, Internet-Nutzung, Medienkompetenz, Lernort, Lernzeit und Finanzierung. Anschließend, ganz der Logik ihrer Frage nach den Wechselwirkungen folgend, präsentiert sie die Typen hinsichtlich der jeweils präferierten und realisierten Netzwerke. Für beide Blickweisen resümiert sie die Konstitutionsleistungen im jeweiligen Typ.

Für die erwachsenenpädagogische Netzwerkforschung ist hier vor allem interessant, wie die Autorin das Verhältnis des „Ausgangsnetzwerks“ (vor Beginn des Online-Lernens) mit dem „Lernnetzwerk“ (also im Online-Lernen) charakterisiert. Gelingt eine Integration zu einem ,erweiterten“ Netzwerk, setzt dies Impulse für weiteres Lernen. Gelingt sie nicht, steht ein Wechsel der Lerngruppe oder ein Abbruch des Lernens ins Haus. Es ist erhellend, die Gruppe der Lernenden ebenso wie das soziale Umfeld der einzelnen Lerner als Netzwerke zu sehen, deren Zusammenwirken oder dessen Gegenteil unterstützend oder behindernd von erheblicher Bedeutung ist.

Müßig zu erwähnen, dass Lauber-Pohle die beiden Seiten der Wechselwirkung, das Online-Lernen und die sozialen Netzwerke, auch theoretisch und in Bezug auf den Forschungsstand ausführlich einleitet. Dies ist besonders in Bezug auf die Netzwerkforschung bemerkenswert, der bis auf ihre Anfänge hin nachgespürt wird. Und von besonderer Qualität ist es, dass die formulierte Fragestellung systematisch und logisch in der Präsentation aufgefächert und präzise beantwortet wird.

Bei allem positiv Anzumerkenden bleibt gelegentlich der Eindruck, dass der eigentliche Prozess des Lernens hinter den technischen Elementen der Online-Angebote und den sozialen Elementen der Netzwerke zu kurz kommt. „Didaktik“ etwa 
wird immer wieder erwähnt, gewinnt aber keine strukturierende Rolle. Das wäre sicher Gegenstand weiterer Untersuchungen, die Lauber-Pohle am Ende ihres Buches anregt. Der Zusammenhang der Online-Lernformen und der sozialen Einbettung der Lerner gibt dies auf jeden Fall her.

\section{Lorenz Lassnigg: Walter Schöni (2017). Bildungswertschöpfung: Zur politischen Ökonomie der berufsorientierten Weiterbildung. Bern: Hep. 284 Seiten, 32,00€ ISBN-Print: 978-3-0355-0733-1}

Gegenüber den gewohnten Texten zur politischen Ökonomie betont Schöni die bildungstheoretische Seite und fasst Bildung in kapitalistischen Gesellschaften grundsätzlich widersprüchlich: als Entwicklung (getrieben von den Lernmotiven der Teilnehmenden), und als Geschäft (getrieben vom Kommerzstreben der Anbietenden). Dieser Widersprüchlichkeit gerecht zu werden, ohne eine der beiden Seiten wegzuschieben, ist ein Grundanliegen des Buches. Wertschöpfung wird nicht nur mittels bildungsökonomischer Wertgrößen behandelt, sondern die (ökonomisch oft verpönte) Seite der Gebrauchswerte wird einbezogen und sowohl betriebswirtschaftlich als auch sozialwissenschaftlich analysiert.

Der Frage, inwieweit man die Wertschöpfung durch Bildungsdienstleistungen auch jenseits der Marktprozesse fassen kann, wird auf verschiedenen Ebenen und multiperspektivisch nachgegangen. Das ist ein großer Anspruch, dem das Buch in einem sehr klaren und übersichtlichen Aufbau mit pointierten Einführungen und Zwischenzusammenfassungen nachkommt.

In Teil 1 wird die berufsorientierte Weiterbildung der Schweiz im Zusammenspiel von Arbeitsmärken und Weiterbildungsangebot untersucht und die gesellschaftliche Wirksamkeit eingeschätzt. Ein Fazit: Der Glaube an den Markt verdeckt grundlegende Defizite.

Teil 2 nutzt betriebswirtschaftliche Theoriestränge für die Grundlagen der Bildungswertschöpfung und wendet vorhandene Wertschöpfungsansätze auf Weiterbildung als Dienstleistung an. „Bildungswerte entstehen [...] nur in Bezugssystemen“ (S. 23). Dieser Hauptteil schließt mit einem Leitfaden zur ,erweiterten Wertschöpfungsanalyse“.

In Teil 3 geht es um operative Fragen der Leistungsprozesse (Prozessorganisation, Controlling). Dabei wird die kooperative Schaffung von Ergebnissen betont. „Vom Anbieter einseitig festgelegte [...] Leistungsprozesse ermöglichen eine glatte Inszenierung, sie schaffen aber nicht unbedingt Outcomes [...]“ (S. 24).

Teil 4 führt die verschiedenen Stränge unter den Stichworten „Märkte“, „Diskurse“ und „Politik“ in einem polit-ökonomischen Ansatz zusammen und konfrontiert Marketing mit Wertschöpfung. ,Wenn das Weiterbildungsmarketing die Wahrnehmung der Wertgrößen bearbeitet, so macht dies das Leistungsangebot nicht wirksamer“ (S. 25). Fazit: Die Marktergebnisse müssen durch bildungswissenschaftliche Analyse und Bewertung ergänzt werden, die für explizite politische Aushandlungen zu nutzen ist. 
Die Bemühung des Autors zielt darauf, in die vielen „Black-Boxes“ der betroffenen Teilbereiche hineinzuleuchten, und die - ansonsten oft abstrakt modellierten - Vorgänge in ihre Bestanteile zu zerlegen. Ein Beispiel betrifft das Zusammenspiel von Angebot und Nachfrage in der Analyse der gesellschaftlichen Wirksamkeit (Kap. 3), wo politische Orientierungsdefizite aufgrund der Informationsmängel auf der Bedarfsseite herausgearbeitet werden. Ein weiteres die Frage, wie durch Bildungsdienstleistungen Werte geschaffen werden (Kap. 5 und 6), wie die Bewertungen der Gebrauchswert-Seite mit den monetären Bewertungen zusammenhängen bzw. wie die einzelwirtschaftliche Wertschöpfung (Anbieter/Kunden) in die breiteren Wertschöpfungs-Kontexte Arbeitsmarkt (symbolische Strukturen), Personalstrategien (Verwertungsregeln) und Standortwettbewerb (Indikatoren, Rankings) eingebettet ist und wie Konflikte und Widersprüche (Parallelsysteme, Verdrängungswettbewerb, Opportunismus gegenüber hegemonialen Diskursen) entstehen. Im vorgeschlagenen qualitativen Konzept der erweiterten Wertschöpfungsanalyse (Kap. 7) wird (als Alternative zu den quantitativen Renditeschätzungen) auf die Kohärenz der Wertschöpfung von der einzelwirtschaftlichen bis zur gesellschaftlichen Ebene fokussiert.

Das Buch trägt zum Diskurs über Inwertsetzungspraktiken ${ }^{1}$ und die Förderung des öffentlichen Gutes bei und leistet einen Beitrag durch die produktive Nutzung der betriebswirtschaftlichen Zugänge in einem breiteren multiperspektivischen Diskurs. Es legt den Fokus nicht auf den monetären Geschäftserfolg sondern auf die Schaffung von Gebrauchswerten und demonstriert anschaulich, in welche ausgedehnten System-Architekturen man gelangt, wenn man die reduktionistische abstrakte Markt-Rhetorik verlässt. Ein zentraler Punkt der Wertschöpfungsanalyse betrifft das Konzept der Äquivalenz (z. B. Preis/Leistung, Tauschwert/Gebrauchswert), das „objektiv" scheint, aber diskursiv-symbolisch (u. a. durch Marketing) aufgebaut werden muss - Informationsnachteile der Nachfrage sind dabei entscheidend.

Das Buch transzendiert, unterstützt durch empirisches Material, die neoliberale Marktrhetorik und bietet praktische Ansätze für die Weiterbildung sowie Argumentationen für Gebrauchswert fördernde Politik. Es kann auch als Inspiration für die Analyse anderer Länder dienen.

\footnotetext{
1 Vgl. Dietzen, A.; Powell, J. J. W., Bahl, A., Lassnigg, L. (Hg.) (2015). Soziale Inwertsetzung von Wissen, Erfahrung und Kompetenz in der Berufsbildung. Weinheim und Basel: Beltz-Juventa.
} 